\title{
Power dissipation in nanoscale conductors: classical, semi-classical and quantum dynamics
}

\author{
Andrew P. Horsfield ${ }^{1}$, D. R. Bowler ${ }^{1,2}$, A. J. Fisher ${ }^{1,2}$, \\ Tchavdar N. Todorov ${ }^{3}$, and M. J. Montgomery ${ }^{3}$ \\ ${ }^{1}$ Department of Physics and Astronomy, University College London, Gower \\ Street, London WC1E 6BT, United Kingdom \\ ${ }^{2}$ London Centre for Nanotechnology, University College London, Gower St, \\ London WC1E 6BT \\ ${ }^{3}$ School of Mathematics and Physics, Queen's University of Belfast, Belfast \\ BT7 1NN, United Kingdom \\ E-mail: a.horsfield@ucl.ac.uk, david.bowler@ucl.ac.uk, t.todorov@qub.ac.uk
}

\begin{abstract}
Modelling Joule heating is a difficult problem because of the need to introduce correct correlations between the motions of the ions and the electrons. In this paper we analyse three different models of current induced heating (a purely classical model, a fully quantum model, and a hybrid model in which the electrons are treated quantum mechanically and the atoms are treated classically). We find that all three models allow for both heating and cooling processes in the presence of a current, and furthermore the purely classical and purely quantum models show remarkable agreement in the limit of high biases. However, the hybrid model in the Ehrenfest approximation tends to suppress heating. Analysis of the equations of motion reveals that this is a consequence of two things: the electrons are being treated as a continuous fluid; and the atoms cannot undergo quantum fluctuations. A means for correcting this is suggested.
\end{abstract}

Submitted to: J. Phys.: Condens. Matter 


\section{Introduction}

Nanoscale devices are of interest both because of the novel phenomena they display and because of the potential practical applications in very high density electronic devices. When we consider these devices, we are often interested in electron transport through them. There has already been substantial work carried out to understand how steady currents flow through these devices, and what forces on the atoms these currents induce[1, 2, 3, 4, 5, 6, 7].

While these calculations provide much useful information about the transport properties of nanoscale devices, there are important questions that they cannot address, notably: what is the Joule heating produced by the current? It is this question that interests us here. To answer it we need to know how the electronic and atomic dynamics are coupled. This coupling has been investigated widely in a number of other contexts, such as non-radiative transitions[8] and photodissociation[9, 10, 11], and many methods of varying complexity and ingenuity have been developed. The range of methods is a testament to the fact that it is exceedingly difficult to obtain good results using available computer resources, except for model problems.

The particular problem that we are interested in poses the following requirements:

(i) To have a current flowing we need open boundary conditions.

(ii) To model the heating of a device we need to consider many non-equivalent atoms and hence many independent degrees of freedom.

(iii) To observe heating, the non-adiabatic coupling between the nuclear and electronic subsystems is essential.

There have been two approaches recently proposed in the literature. The first one makes use of perturbation theory to couple quantum atoms to current carrying electrons $[12,13,14]$, while the second one uses non-adiabatic molecular dynamics to couple classical atoms to current carrying electrons[15]. These two approaches are very different, and the purpose of this paper is to analyse and compare them, together with a simple classical model. In the remainder of this paper we will summarise the theory for each approach, and characterise the important results it produces. We begin with a purely classical model which reproduces some of the qualitative features of the other models, and has the merit of great transparency.

\section{Classical model}

In the first model that we consider, we treat both electrons and atoms as classical particles. In a metallic wire a current is carried only by a small number of electrons within a narrow energy window near the Fermi level. The remaining very much larger number of electrons contribute to the binding of the atoms. Thus in our classical model we represent our wire as charge neutral atoms bound to sites by springs (corresponding to a nucleus plus the electrons that are associated with it and its bonds to neighbours), and a low-density of independent current carrying electrons travelling down the wire. We consider the scattering of the current carrying electrons by the atoms to be random and instantaneous events. The fact that the events are random allows us to ignore correlations between scattering events. The fact that the scattering is instantaneous allows us to ignore the details of the atomic trajectories. We also assume that the motion occurs in one dimension only. First we derive some simple analytic results. We follow this with more detailed computer simulations. 


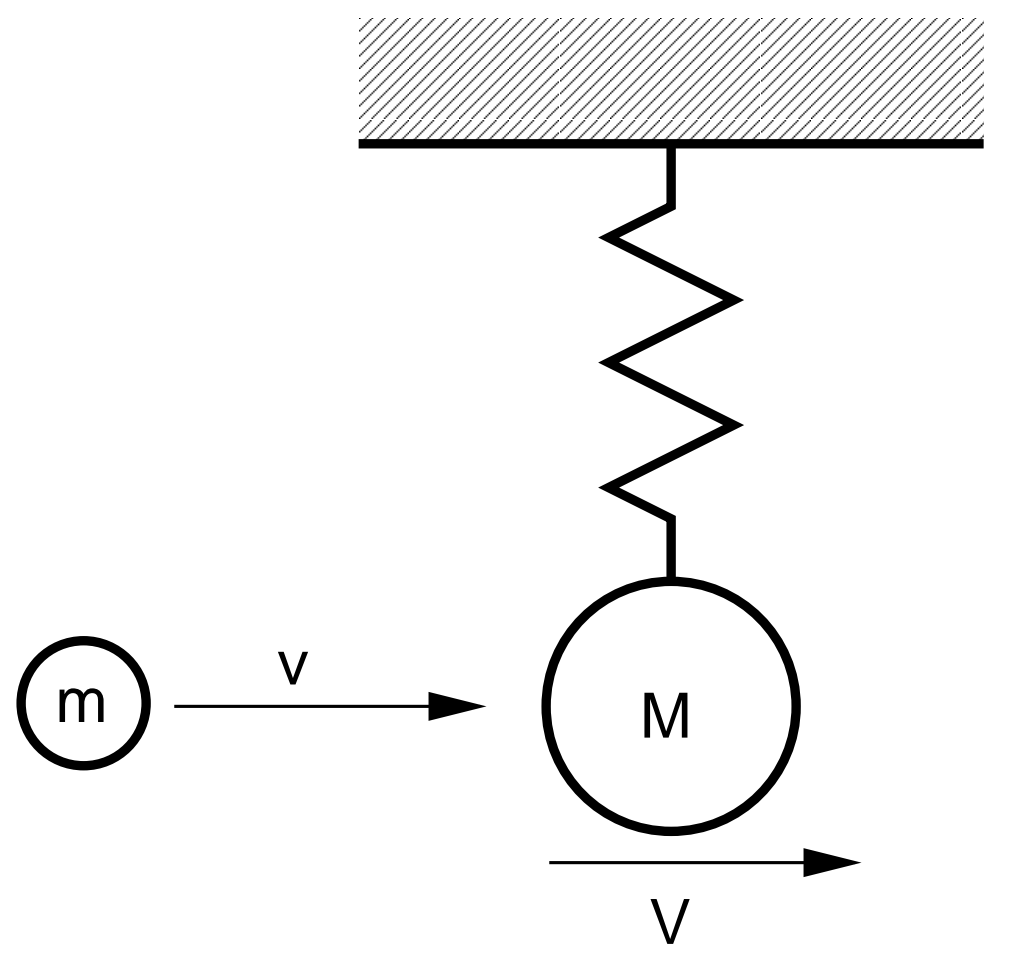

Figure 1. A schematic representation of the initial configuration for a classical electron (with mass $m$ ) about to collide with a classical atom (with mass $M$ ). The electron and atom have initial velocities $\mathrm{v}$ and $\mathrm{V}$, respectively. The atom is anchored by a spring, and oscillates about its equilibrium position.

\subsection{Analytical Treatment}

The velocities of a current carrying electron and an atom immediately before a collision are shown in Fig. 1. By conserving kinetic energy and linear momentum, making use of the fact that the electron is much lighter than the atom $(m<<M)$, and averaging over all possible initial atomic velocities, of both signs, for the average change in kinetic energy of the atom in a collision we get $\Delta K_{\text {atom }} \approx 4(m / M)\left(K_{e}-K_{\text {atom }}\right)$. Here, $K_{e}=m v^{2} / 2$ and $K_{\text {atom }}=M\left\langle V^{2}\right\rangle / 2$. If we furthermore allow for the slight difference in average collision frequency under positive and negative $V$, for a given $v$, and multiply by the incident electron current, then for the average net power $(w)$ delivered to the atom we obtain

$$
w \approx 4 j\left(\frac{m}{M}\right)\left(K_{e}-2 K_{\text {atom }}\right)
$$

where $j$ is the mean incident electron particle current. We see that the power is composed of two competing terms. In the limit where the excess energy of the electrons (the energy that can be accessed in inelastic collisions), $K_{e}$, much exceeds the average atomic kinetic energy, $K_{\text {atom }}$, the heating term dominates and we are left with

$$
w \approx 4 j\left(\frac{m}{M}\right) K_{e} .
$$

A key feature of this result is that the net power transferred to the atom is positive. That is, a current leads to heating. The larger the current $(j)$, the greater the rate 
of heating. However, if we imagine smearing the discrete classical electrons out into a continuous fluid $(m \rightarrow 0)$ with the same mass flux $(j m)$, then $K_{e} \rightarrow 0$ and the cooling term is all that we are left with,

$$
w \approx 4 j\left(\frac{m}{M}\right)\left(-2 K_{\text {atom }}\right) .
$$

We will return to these results in Section 5.

\subsection{Computer Simulations}

We now study the above classical model by a computer simulation. The atom attached to the spring oscillates freely, except when undergoing instantaneous collisions with an electron. The result of the collision is to change the velocity of the atom, the change being calculated by conserving energy and linear momentum

$$
\Delta V=\frac{2(v-V)}{(M / m)+1}
$$

The change in velocity results in a change in amplitude of oscillation between collisions. The velocity of the incoming electrons is kept constant (corresponding to the Fermi velocity), but the time between collisions is varied corresponding to different currents and rates of scattering of the electrons by the atom. It was found to be important to introduce random variations in the times between collisions, otherwise the highly correlated sequence of scattering events would produce cooling instead of heating. For a particular atom, the average time between collisions $\left(\bar{t}_{c}\right)$ is calculated from $\bar{t}_{c}=\tau_{D} / Z$, where $\tau_{D}$ is the relaxation time in the Drude equation for conductivity, and $Z$ is the average number of current carrying electrons per atom. Because the original Drude formula (in terms of which we define our parameters) fails to take Fermi statistics into account, we adjust the relaxation time to hold the conductivity constant as we vary number of current carrying electrons according to $\tau_{D}=\tau_{D}^{(0)} Z^{(0)} / Z$, where $Z^{(0)}$ is the number of valence electrons per atom, and $\tau_{D}^{(0)}$ is the standard Drude relaxation time. For our numerical simulations we use values for aluminium $\left(Z^{(0)}=3\right.$, $\tau_{D}^{(0)}=8 \mathrm{fs}, M=27 \mathrm{amu}, \omega=0.013 / \mathrm{fs}, v=20.3 \AA /$ fs and $\left.Z / Z^{(0)}=0.002\right)$. The number of carriers $\left(Z / Z^{(0)}\right)$ we are using is very low, leading to low rates of heating, but this does not change the conclusions. Typical results for the variation of the kinetic energy of one atom as a function of time are shown in Fig. 2. We see that the atom undergoes a rise in its kinetic energy (heating) with increasing time.

For the discussion so far we have, quite correctly, treated the electrons as discrete particles. However, it will be important to understand how the heating behaviour changes as we move from a discrete description to a continuum one when we come to investigate the Ehrenfest approximation. We make this transition in our simulations by dividing each electron into a number of smaller pieces. Suppose we divide one electron into $S$ pieces, to maintain the same current we reduce the time between collisions by this same factor. Referring to Fig. 2 we see that the heating disappears rapidly as we increase the number of pieces into which the electron has been subdivided. By the time the electron has been divided into 100 pieces we get steady slow cooling. This is consistent with the analytic results obtained above.

We performed one further set of calculations in which the electrons are once again sliced up into pieces, but these pieces are bunched up in space to form compressed packets with empty space between them. This roughly corresponds to travelling packets of fluid. From Fig. 3 we see that tight packets behave essentially the same 


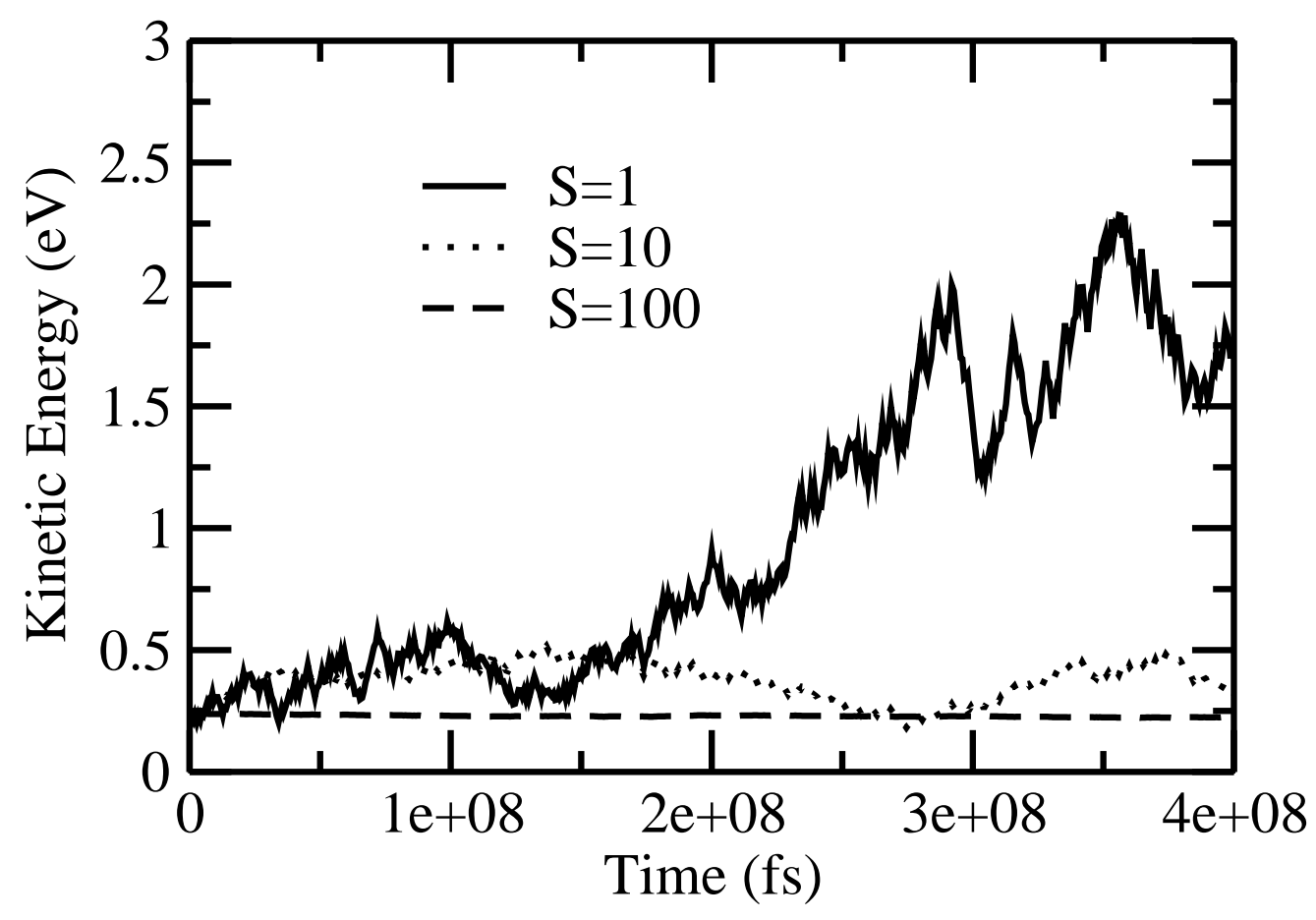

Figure 2. This graph shows the variation of the kinetic energy of the atom as a function of time. The three curves correspond to the different number of pieces (S) into which the electron has been chopped.

as discrete particles. This is not surprising, but it tells us that highly localised disturbances in a fluid can behave like discrete particles. We will elaborate on this in the discussion of the Ehrenfest method.

\section{Quantum Perturbation Theory}

The physical model used to develop the perturbation theory expression for the interaction of vibrating ions immersed in a sea of current carrying electrons $[12,13,14]$ is an extension of the above classical model. Initially we imagine the ions to be immersed in the electron gas in its ground state. We then excite the electrons by causing them to carry a current, and once this is established we study the response of the ionic motion to this excitation of the electron gas.

First, we describe the general setup for the perturbative treatment of the interaction between quantum vibrations and excited quantum electrons. To describe the unperturbed state of the system we make the Born-Oppenheimer separation and solve the adiabatic equations. The solution of these equations describes oscillatory motion of the ions about equilibrium fixed sites. Next, we consider the excited states of the electrons in the presence of static ions at their equilibrium sites, and place the electrons in one such excited state. Finally, we couple the electronic and ionic subsystems together and calculate the resultant energy transfer between them. This 


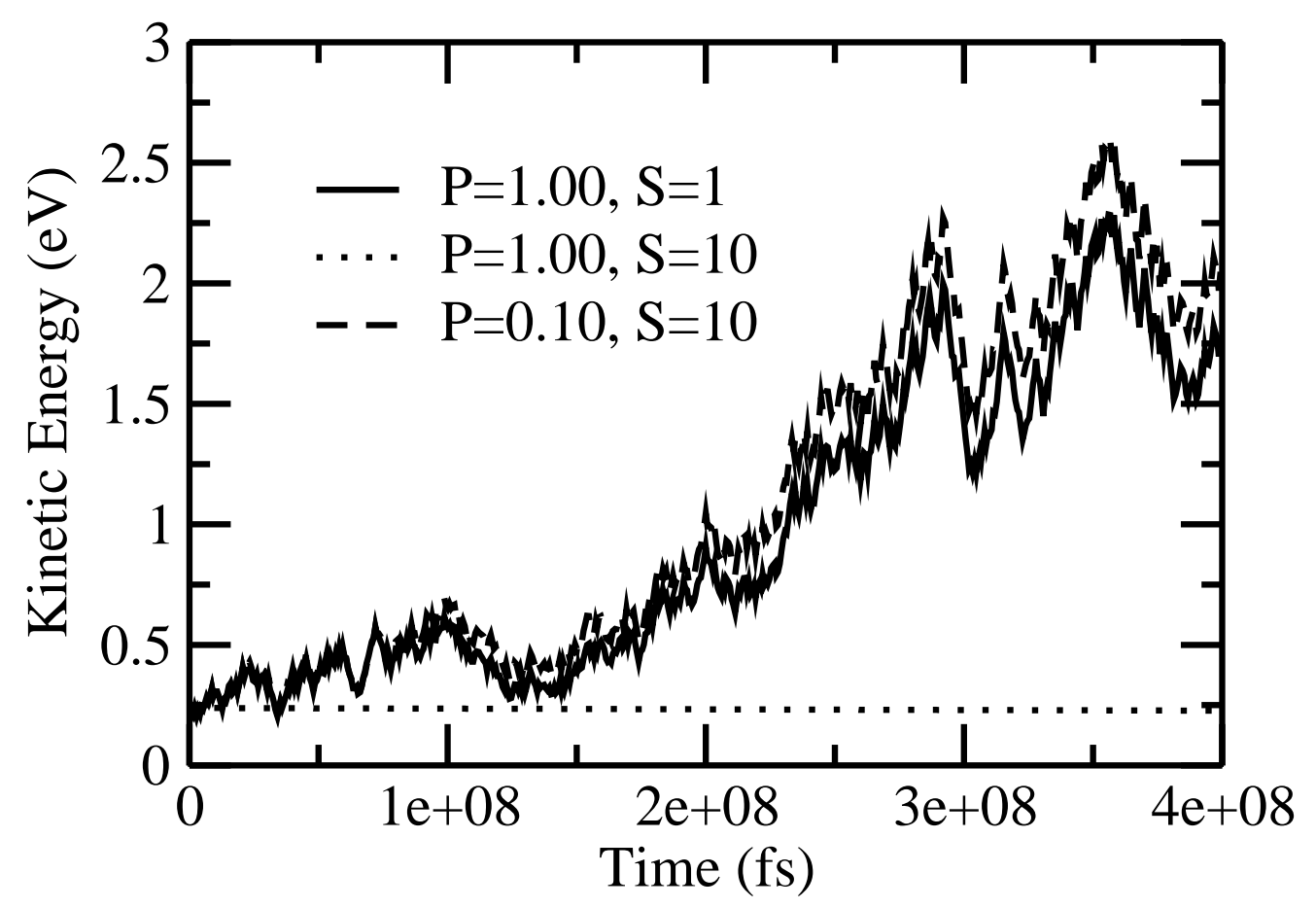

Figure 3. This graph shows the variation of the kinetic energy of the ion as a function of time. Each curve is characterised by two quantities: the number of pieces into which the electron has been sliced $(\mathrm{S})$, and the width of the packet (P) created from a sliced electron. The width of a packet is defined as the ratio of the following two quantities: the time difference between the collision for the first piece and last piece of the electron; the period of oscillation of the atom. The case $S=1$ corresponds to an intact electron.

procedure corresponds to the following partitioning of the Hamiltonian for the system:

$$
\begin{aligned}
\hat{H}= & \sum_{I} \hat{T}_{I}+\hat{H}_{e}(\vec{R}) \\
= & {\left[\sum_{I} \hat{T}_{I}+E_{0}(\vec{R})-E_{0}\left(\vec{R}_{0}\right)\right]+\hat{H}_{e}\left(\vec{R}_{0}\right)+} \\
& {\left[\left(\hat{H}_{e}(\vec{R})-E_{0}(\vec{R})\right)-\left(\hat{H}_{e}\left(\vec{R}_{0}\right)-E_{0}\left(\vec{R}_{0}\right)\right)\right] } \\
= & \hat{H}_{I}+\hat{H}_{e}\left(\vec{R}_{0}\right)+\hat{H}_{e I}
\end{aligned}
$$

where $\vec{R}$ represents the ionic coordinates, $\vec{R}_{0}$ represents the equilibrium fixed sites, $\hat{T}_{I}$ is the ionic kinetic energy operator for one ion and $\hat{H}_{e}(\vec{R})$ is the electronic Hamiltonian in the field of static ions. The ion-ion interaction is included in $\hat{H}_{e}(\vec{R}) . E_{0}(\vec{R})$ is the total energy for the ground state Born-Oppenheimer surface.

The initial state of the ionic subsystem is a solution of the equation

$$
\hat{H}_{I}\left|\chi_{N}\right\rangle=U_{N}\left|\chi_{N}\right\rangle \text {. }
$$

The energies $U_{N}$ correspond to the phonon spectrum in the harmonic approximation. The excited states of the electrons, in the field of static ions in their equilibrium 
positions, are solutions to the Born-Oppenheimer equation

$$
\hat{H}_{e}(\vec{R})\left|\Phi_{n}\right\rangle=E_{n}(\vec{R})\left|\Phi_{n}\right\rangle
$$

with $\vec{R}=\vec{R}_{0}$.

We can now couple the two subsystems together. We expand the states of the coupled system as a linear combination of products of the two sets of eigenstates. Let us define $\langle\vec{r} \vec{R} \mid n N\rangle=\left\langle\vec{r} \mid \Phi_{n}\right\rangle\left\langle\vec{R} \mid \chi_{N}\right\rangle$. The coefficients in the expansion are found using perturbation theory. The quantity we are interested in is the heating of the ions. The operator corresponding to the rate of heating is $\left[\hat{H}_{I}, \hat{H}_{e I}\right] / \mathrm{i} \hbar$. If the system starts in state $|n N\rangle$, then the lowest order contribution to the expectation value of this operator is

$$
w=\frac{2 \pi}{\hbar} \sum_{n^{\prime} N^{\prime}}\left|\left\langle n^{\prime} N^{\prime}\left|\hat{H}_{e I}\right| n N\right\rangle\right|^{2}\left(U_{N^{\prime}}-U_{N}\right) \delta\left(E_{n}+U_{N}-E_{n^{\prime}}-U_{N^{\prime}}\right)(6)
$$

In the limit of small ionic displacements we can use a linear approximation for the interaction Hamiltonian: $\hat{H}_{e I} \approx\left(\vec{R}-\vec{R}_{0}\right) \cdot \vec{\nabla} \hat{H}_{e}\left(\vec{R}_{0}\right)$. Here we have used the definition of the fixed sites, namely $\vec{\nabla} E_{0}\left(\vec{R}_{0}\right)=0$. Matrix elements of this Hamiltonian have the form $\left\langle n^{\prime} N^{\prime}\left|\vec{H}_{e I}\right| n N\right\rangle=\left\langle\chi_{N^{\prime}}\left|\vec{R}-\vec{R}_{0}\right| \chi_{N}\right\rangle \cdot\left\langle\Phi_{n^{\prime}}\left|\vec{\nabla}_{e}\left(\vec{R}_{0}\right)\right| \Phi_{n}\right\rangle$.

We now consider a single dynamical ion with a single vibrational degree of freedom. If this Einstein oscillator has an angular frequency $\omega$ and a mass $M$, then $\left\langle\chi_{N^{\prime}}\left|\vec{R}-\vec{R}_{0}\right| \chi_{N}\right\rangle=\sqrt{\hbar / 2 M \omega}\left[\sqrt{N} \delta_{N^{\prime}, N-1}+\sqrt{N+1} \delta_{N^{\prime}, N+1}\right]$, which follows from expressing the displacement in terms of ladder operators. If we further assume that the electronic wave functions are single Slater determinants constructed from single particle orbitals $|\alpha\rangle$ with eigenvalues $\epsilon_{\alpha}$ and occupancies $f_{\alpha}$, and that $\vec{\nabla} \hat{H}_{e}\left(\vec{R}_{0}\right)=$ $\sum_{i} \vec{g}\left(\vec{r}_{i}\right)$, where $\vec{r}_{i}$ is an electronic coordinate, we get

$$
\begin{aligned}
w=\frac{\pi \hbar}{M} \sum_{\alpha \beta} & f_{\alpha}\left(1-f_{\beta}\right)|\langle\beta|\vec{g}| \alpha\rangle|^{2} \times \\
& \left\{(N+1) \delta\left[\epsilon_{\alpha}-\left(\epsilon_{\beta}+\hbar \omega\right)\right]-N \delta\left[\epsilon_{\alpha}-\left(\epsilon_{\beta}-\hbar \omega\right)\right]\right\} .
\end{aligned}
$$

When $N=0, w \geq 0$, which means that only ionic heating is possible. This is necessarily true as there are no phonon excitations available to be removed to cause ionic cooling. The physical mechanism of the heating in the limit $N=0$ is spontaneous phonon emission: even with the ions in the vibrational ground state, their zeropoint motion produces a finite electron-phonon scattering probability, which results in inelastic decay of the excited electronic state. But this heating is also an example of a more general feature: for a given pair of states the heating transition will be faster than the cooling transition because $N+1>N$.

Let us now turn to the case of a current carrying nanoscale conductor. To set up current flow, we imagine that the conductor of interest is connected to two semi-infinite metallic electrodes, forming an open-boundary conducting system. The one-electron states $|\alpha\rangle$ for this system can be chosen as current carrying scattering eigenstates, divided into two classes [7]. The states in one class, $\left|\alpha_{1}\right\rangle$ with energies $\epsilon_{1}$, consist of a right-travelling wave, incident in the left electrode upon the junction, then partially reflected back into the left electrode and partially transmitted into the right electrode, and conversely for the other class, $\left|\alpha_{2}\right\rangle$ with energies $\epsilon_{2}$. We introduce the partial density of states operators

$$
D_{1}(E)=\sum_{1}\left|\alpha_{1}\right\rangle \delta\left(E-\epsilon_{1}\right)\left\langle\alpha_{1}\left|, \quad D_{2}(E)=\sum_{2}\right| \alpha_{2}\right\rangle \delta\left(E-\epsilon_{2}\right)\left\langle\alpha_{2}\right| .(8)
$$


$D_{1}(E), D_{2}(E)$ and the total density of states operator $D(E)=D_{1}(E)+D_{2}(E)$, can be expressed in terms of the Green's function for the electrode-junction-electrode system $[13,7]$.

To set up current flow we imagine that a battery of voltage $W$ populates the states $\left|\alpha_{1}\right\rangle$ and $\left|\alpha_{2}\right\rangle$ with occupancies $f_{1}(E)$ and $f_{2}(E)$, where $f_{1}(E)$ and $f_{2}(E)=$ $f_{1}(E+e W)$ are Fermi-Dirac distribution functions with electrochemical potentials $\mu_{1}$ and $\mu_{2}=\mu_{1}-e W$, respectively [7]. For simplicity, we consider the case of zero electronic temperature, where $f_{1}(E)$ and $f_{2}(E)$ are step functions, offset by an amount $e W$.

We consider the simple scenario in which our conducting system is a onedimensional atomic wire, and our Einstein oscillator is one particular ion in it, with label $s$ and position $R_{s}$, free to execute simple harmonic vibrations along the axis of the wire. Generalisations of the quantum perturbative treatment to systems with multiple vibrational degrees of freedom and beyond the Einstein independent-oscillator approximation are given in Refs. $[13,14,16]$. We adopt a single-orbital nearestneighbour tight-binding model. The one-electron operator $g$ in Eq. (7) then takes the form $[13,16]$

$$
g=\sum_{s^{\prime} \neq s}\left(|s\rangle\left\langle s^{\prime}|+| s^{\prime}\right\rangle\langle s|\right) \frac{\partial H_{s s^{\prime}}}{\partial R_{s}}
$$

where $|s\rangle$ is the tight-binding basis state at site $s$ and $H_{s s^{\prime}}$ are the hopping integrals between $s$ and neighbouring sites $s^{\prime}$. The derivatives $\partial H_{s s^{\prime}} / \partial R_{s}$ are evaluated at the equilibrium atomic positions.

We make the following mild further approximations. We assume that electronic properties, and the electron Green's functions in particular, do not vary significantly over energies of the order of $\hbar \omega$. We assume also that we are in the linear voltage regime, where the electron Green's functions do not vary significantly over the energy window for conduction, between $\mu_{2}$ and $\mu_{1}=\mu_{2}+e W$. Then Eq. (7) for the power into our oscillator reduces to [13]

$$
w \approx-\frac{2 \pi \hbar}{M} U\left(T_{11}+2 T_{12}+T_{22}\right)+\frac{2 \pi \hbar}{M}(|e W|-\hbar \omega) \theta(|e W|-\hbar \omega) T_{12}(10)
$$

where $U=N \hbar \omega$ is the thermal energy of the oscillator, $T_{i j}=\operatorname{Tr}\left[D_{i} g D_{j} g\right]$ for $i, j=1,2$, and $D_{1}$ and $D_{2}$ are evaluated at the Fermi level, in the absence of the current. $\theta$ is the step function defined by $\theta(x)=0$ for $x<0, \theta(x)=1$ otherwise. Eq. (10) includes a factor of 2 for spin degeneracy.

The appearance of the step function $\theta$ in Eq. (10) is a consequence of the need to satisfy simultaneously the Pauli exclusion principle and energy conservation. Processes in which an electron excites the oscillator, by generating a quantum of vibrational energy of $\hbar \omega$, are possible only if there is an empty electronic state with a correspondingly lower energy for the electron to go into. This requires the excess electron energy, set by the bias $W$, to be larger than $\hbar \omega$, for any heating of the ion to occur. Thus, for $|e W|<\hbar \omega$, only cooling processes are possible and $w<0$. At $|e W|=\hbar \omega$, heating processes are first switched on. Upon increasing the bias further, the second term in Eq. (10) will begin to dominate, resulting in net heating of the ion. This net heating is a manifestation of the overall dominance of the rate of heating processes over the rate of cooling processes that became apparent already at the level of Eq. (7), and is consistent also with the classical model, discussed earlier.

To make a direct comparison with that classical model, we now consider the simplest possible case, in which our one-dimensional conductor is a perfect uniform 
linear chain, with one-electron states $|\alpha\rangle$ that are one-dimensional Bloch waves. Then Eq. (10) reduces to

$$
w \approx \frac{2 \pi \hbar}{M} \frac{1}{\pi^{2}} \frac{H^{\prime 2}}{H^{2}}[-2 U+(|e W|-\hbar \omega) \theta(|e W|-\hbar \omega)]
$$

where $H$ and $H^{\prime}$ are the equilibrium nearest-neighbour hopping integral and its derivative with distance. As in Eq. (1), the power $w$ into our ion in Eq. (11) is made up of two competing terms. One, with a negative sign corresponding to ionic cooling, is set by the vibrational energy of the ion ( $K_{\text {atom }}$ in Eq. (1) and $U$ above). The other, corresponding to heating, is set by the excess energy of the electrons $\left(K_{e}\right.$ in the classical case in Eq. (1) and $e W$, corrected by an amount $\hbar \omega$ due to the fermionic statistics of the electrons as discussed earlier, in Eq. (11)).

In the limit $|e W| \gg \hbar \omega,|e W| \gg U$ Eq. (11) reduces to

$$
w \approx \frac{2 \pi \hbar}{M} \frac{1}{\pi^{2}} \frac{H^{\prime 2}}{H^{2}}|e W|
$$

This is the analogue of Eq. (2). Let $p_{F}$ be the Fermi momentum of the electrons. Setting $K_{e}=p_{F}^{2} / 2 m, j=e W / \pi \hbar$ (from the Landauer formula for a perfect onedimensional conductor) and $\left|H^{\prime} / H\right| \sim 1 / a$, where $a$ is something of the order of the interatomic distance, for the ratio of the right-hand sides of Eq. (2) and Eq. (12) we find $p_{F}^{2} a^{2} / \hbar^{2} \sim 1$. We conclude that, in the high-bias limit, the classical and quantum power formulae are not only in qualitative but also in approximate quantitative agreement.

\section{The Ehrenfest Approximation}

The Ehrenfest approximation to the time-dependent Schrödinger equation has been derived many times before in a variety of ways (see for example Ref. [17]). But it is very helpful for our later discussion to derive it from a Hamiltonian that is very similar to the one used in the previous section.

We start from Eq. (3), and begin by replacing the reference ionic coordinates with the expectation value of the ionic coordinates $\bar{R}_{I}=\operatorname{Tr}\left\{\hat{\rho} \hat{R}_{I}\right\}$, where $\hat{\rho}$ is the density matrix describing the electron-ion system. For the ionic Hamiltonian $\hat{H}_{I}$ we make a quadratic expansion about the mean coordinate, and for the electron-ion interaction we make a linear expansion. This gives us the following quantum-classical Hamiltonian

$$
\begin{gathered}
\left.\hat{H}_{Q C}=\sum_{I} \hat{T}_{I}+\hat{H}_{e}(\bar{R})-\sum_{I} \hat{X}_{I} \cdot \hat{F}_{e I}(\bar{R})+\frac{1}{2} \sum_{I J} \hat{X}_{I} \cdot K_{I J}(\bar{R}) \cdot \hat{1} \beta\right) \\
\hat{F}_{e I}(\bar{R})=-\frac{\partial \hat{H}_{e}(\bar{R})}{\partial \bar{R}_{I}}, \quad K_{I J}(\bar{R})=\frac{\partial^{2} E_{0}(\bar{R})}{\partial \bar{R}_{I} \partial \bar{R}_{J}}
\end{gathered}
$$

where $\hat{X}_{I}=\left(\hat{R}_{I}-\bar{R}_{I}\right)$. This Hamiltonian would be exactly the one in Section 3 if $\bar{R}$ is independent of time. It is straightforward to derive the following Ehrenfest-like equations from this Hamiltonian:

$$
\begin{array}{ll}
\frac{\mathrm{d} \bar{R}_{I}}{\mathrm{~d} t}=\frac{\bar{P}_{I}}{M_{I}}, \quad \bar{P}_{I}=\operatorname{Tr}\left\{\hat{\rho} \hat{P}_{I}\right\}, \quad \frac{\mathrm{d} \bar{P}_{I}}{\mathrm{~d} t}=\operatorname{Tr}_{e}\left\{\hat{\rho}_{e} \hat{F}_{e I}(\bar{R})\right\} \\
\mathrm{i} \hbar \frac{\partial \hat{\rho}_{e}}{\partial t} \quad=\left[\hat{H}_{e}(\bar{R}), \hat{\rho}_{e}\right]-\sum_{I}\left[\hat{F}_{e I}(\bar{R}), \hat{\mu}_{I}\right] \\
\hat{\rho}_{e}=\operatorname{Tr}_{I}\{\hat{\rho}\}, \quad \hat{\mu}_{I}=\operatorname{Tr}_{I}\left\{\hat{X}_{I} \hat{\rho}\right\}
\end{array}
$$


where $M_{I}$ is the mass of ion $I$. These would be exactly the Ehrenfest equations if $\hat{\mu}_{I}=0$. This corresponds to the case in which the ions are unable to deviate from the mean trajectory, and occurs in one of two limits: the mass of the ions becomes infinite; or the second derivatives of the reference energy $\left(K_{I J}(\bar{R})\right)$ becomes infinite. The calculations below show the consequences of ignoring this term for the modelling of the Joule heating of the ions. However, from the quantum perturbation formalism (see Eq. (7)) we can make one prediction. In the limit $K_{I J} \rightarrow \infty$, which corresponds to $\omega \rightarrow \infty$, all transitions are suppressed because quantum fluctuations of the ion about its mean position are suppressed. Clearly therefore the quantum perturbation formalism and the Ehrenfest method will generate heating and cooling by different mechanisms.

\subsection{Ehrenfest simulations of current-carrying systems using a tight-binding electronic Hamiltonian}

In principle we could evaluate the time evolution of the ionic forces directly from Eqs. (15) and (16). However these are many body equations and thus computationally intractable for all but the smallest systems. Since the Ehrenfest equations correspond to electrons in the field of classical ions that create a time varying external potential we can evaluate the electronic properties using time-dependent density functional theory[18, 19]. (The formalism for doing this in the presence of open boundary conditions, which are needed in order to support an electric current, has been described elsewhere[15].) This then allows us to use the single particle density matrix. The single particle density matrix will be represented by $\hat{\rho}_{e}^{(1)}$. The relevant equations then are

$$
\begin{aligned}
\mathrm{i} \hbar \frac{\partial \hat{\rho}_{e}^{(1)}}{\partial t} & =\left[\hat{h}_{K S}, \hat{\rho}_{e}^{(1)}\right] \\
\hat{h}_{K S} & =\hat{T}+\hat{v}_{e f f}\left[\hat{\rho}_{e}^{(1)} ; \bar{R}\right] \\
\vec{F}_{I} & =-\operatorname{Tr}_{e}\left\{\hat{\rho}_{e}^{(1)} \vec{\nabla}_{I} \hat{h}_{K S}\right\}-\vec{\nabla}_{I} E_{I I}(\bar{R})
\end{aligned}
$$

where $\hat{h}_{K S}$ is the Kohn Sham Hamiltonian, and $\hat{v}_{\text {eff }}[\hat{\rho}, \bar{R}]$ is the effective Kohn-Sham potential which depends on the electron density (and hence the density matrix) and ionic coordinates. The gradient with respect to ionic coordinates is taken at fixed density. The ion-ion interaction is given by $E_{I I}(\bar{R})$, and $\vec{F}_{I}$ is the force on ion $I$. Note that the trace over electronic degrees of freedom in Eq. (20) includes spin variables.

A current is generated by having a density matrix that corresponds to an excess of electrons on one side of the system and a deficit on the other[19] (for example, the plates of a charged capacitor). This density matrix evolves under the influence of the Hamiltonian (Eq. (18)), which leads to a flow of electrons from the region where there is an excess to the region where there is a deficit, producing an electric current.

For this work we consider an infinite chain of atoms, with the electronic structure represented by a simple tight binding model with one s orbital per atom. The middle atom has an increased on-site energy $(1 \mathrm{eV})$ to act as a barrier to current flow. This atom we term the device, and is the only atom that we allow to move. The hopping integral between neighbouring atoms is given by $h(r)=h_{0}\left(r_{0} / r\right)^{2}$, where $h_{0}=-1 \mathrm{eV}$ and $r_{0}=1 \AA$, and the repulsive term is given by $\phi(r)=\phi_{0}\left(r_{0} / r\right)^{4}$ with $\phi_{0}=0.6 \mathrm{eV}$.

Results of simulations are shown in Fig. 4 where the kinetic energy of the dynamic atom is plotted as a function of time. From this plot we can see several features:

(i) As we increase the bias the system moves from undergoing cooling to undergoing heating. 


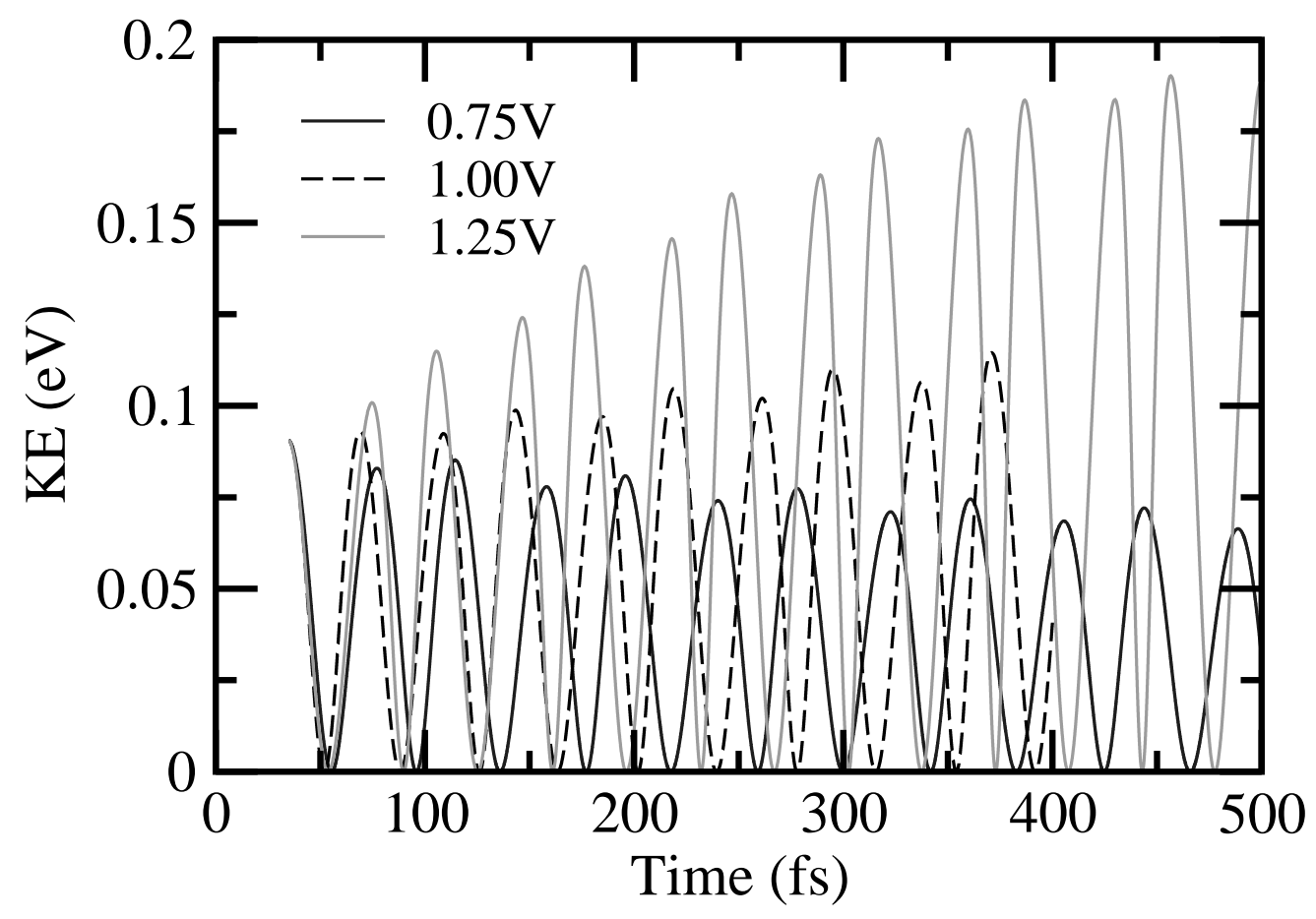

Figure 4. The variation of kinetic energy of the dynamic atom as a function of time for a range of applied biases. The applied bias in volts is indicated by the style of the line (see the legend). The frequency of oscillation is $\omega=8.4 \times 10^{13} \mathrm{~s}^{-1}$, and hence $\hbar \omega=0.055 \mathrm{eV}$.

(ii) After sufficiently long times the system appears to reach an equilibrium.

Qualitatively, this is clearly correct. For very low biases $(\mathrm{e} W<\hbar \omega=0.055 \mathrm{eV}$, where $W$ is the bias and $\omega$ is the frequency of oscillation of the atom) the only electronic transitions that can be induced by the oscillating atom are excitations to higher energies. In this case, the oscillating atom will undergo cooling. For larger biases, electrons can make downward transitions, providing energy to the oscillating atom, producing heating. The greater the bias, the greater the amount of heating expected, because downward transitions should occur more quickly than upward ones[12].

However, a closer examination of the numbers shows that there are some problems. Even with a fairly large bias of $0.75 \mathrm{~V}$ we find cooling. With a bias of this size $(|e W| \gg \hbar \omega,|e W| \gg U)$, we would definitely expect heating (see Eq. (12)). To understand why cooling is taking place, we need to look at the Ehrenfest approximation in an appropriate way. From Eq. (18) we see that the electrons are being characterised by their average charge density, and so can be thought of as a fluid[20] responding to an external time varying classical field generated by the moving ions. Similarly, from Eq. (20) we find that the ions are responding to the classical field generated by this fluid of electrons (only the average charge density appears in the force equation). So we find that the Ehrenfest equations are describing the interaction between point like classical ions and an electron fluid. From our classical simulations above we discovered that turning the electrons into a fluid resulted in the suppression 
of heating.

So we now have an explanation for the cooling. But this leaves us with a new problem: why do we ever see heating? Once again the answer can be found from the classical simulations. There we found that if we treat the electrons as a fluid, but instead of being uniform it propagates in packets, heating is once more observed. The tighter the packets, the closer the result to that corresponding to point like electrons. In our Ehrenfest simulations, the oscillating atom introduces fluctuations into the electron density. These in turn can couple to the oscillating atom, and thereby introduce heating.

To obtain the correct heating behaviour we need to reintroduce the full expression for the evolution of the electrons given in Eq. (16). Note that we are returning to the many-body electron density matrix, not the single particle one used above. This is not essential, but reducing Eq. (16) to a single particle form involves further manipulations that add nothing to the present discussion. The second term, which is lost in the Ehrenfest approximation, introduces correlations between individual electrons and the ions. To understand why this is, we need to look at the structure of the moment operator $\hat{\mu}_{1}$. This is most transparent if we look at diagonal matrix elements of the operator in a spatial representation

$$
\begin{array}{r}
\mu_{1}\left(\vec{r}_{1} \ldots \vec{r}_{n} ; \vec{r}_{1} \ldots \vec{r}_{n}\right)=\int \mathrm{d} \vec{R}_{1} \ldots \mathrm{d} \vec{R}_{N}\left(\vec{R}_{I}-\vec{R}_{I}\right) \times \\
\rho\left(\vec{R}_{1} \ldots \vec{R}_{N}, \vec{r}_{1} \ldots \vec{r}_{n} ; \vec{R}_{1} \ldots \vec{R}_{N}, \vec{r}_{1} \ldots \vec{r}_{n}\right)
\end{array}
$$

For a pure state these diagonal elements of the full density matrix just correspond to the modulus squared of the wave function. Therefore, we can interpret the moment as being the mean displacement of an ion from the average trajectory given that the electrons have positions given by $\vec{r}_{1} \ldots \vec{r}_{n}$. That is, this moment has explicit information correlating the positions of individual ions with positions of individual electrons. This means we are no longer treating the electrons as a fluid, and thus would expect to observe correct heating behaviour once this term is included. It also introduces quantum fluctuations of the ions. This is currently being investigated, and will be the subject of a future paper.

\section{Noise Analysis}

We may gain further insight into the microscopic origin of current-induced ionic heating, and into the nature of the Ehrenfest approximation, by the following analysis in terms of fluctuations[21]. Consider a single classical vibrating ion of mass $M$ in a one-dimensional current-carrying atomic wire between macroscopic electrodes held under a given bias. There are two interesting types of fluctuation in this system: the displacement of the ion from its mean position; the variation in the electron density (for a fixed set of ionic positions) about the value determined for the steady state.

Note that, although the equations below are written down for the Ehrenfest model, they apply equally to the classical model of Section 2. The difference is only in how the force on the ion due to the electrons $\left(F_{e}\right)$ is calculated, as we will see below.

\subsection{Ehrenfest Approximation}

Let $R=R(t)$ and $P=P(t)=M \mathrm{~d} R / \mathrm{d} t$ be the position and momentum of the ion. In the Ehrenfest equation of motion we can partition the force on the ion into a 
component from the electrons, and a component from the other ions

$$
\frac{\mathrm{d} P}{\mathrm{~d} t}=F=F_{e}+F_{I}
$$

where $F_{e}=F_{e}(t)$ and $F_{I}=F_{I}[R(t)]$ are the forces exerted on the ion by the current-carrying electron gas and by all other ions, respectively. The Ehrenfest force, $F=F_{e}+F_{I}$, is given by equation 15 . We can rewrite equation 22 in terms of fluctuations of the electronic force about the steady state value

$$
\frac{\mathrm{d} P}{\mathrm{~d} t}=\left(F_{e}-F_{0}\right)+\left(F_{I}+F_{0}\right)
$$

where $F_{0}=F_{0}[R(t)]$ is the force that would be exerted on the ion by the currentcarrying electron subsystem if the ion were held rigidly at its instantaneous position $R=R(t)$ and the electrons were in the current-carrying steady state, corresponding to the given voltage and the given instantaneous atomic geometry $[5,3]$.

Let $\Delta R=R-R_{0}$ be the displacement of the ion from the steady state equilibrium position $R_{0}$, and $\Delta F=\Delta F(t)=F_{e}-F_{0}$ be the deviation of the force exterted by the electrons on the ion from the steady-state force $F_{0}=F_{0}[R(t)]$. We can then write Eq. (23) as

$$
M \frac{\mathrm{d}^{2} \Delta R}{\mathrm{~d} t^{2}}=\Delta F-M \omega^{2} \Delta R
$$

We have substituted $F_{I}+F_{0}=-M \omega^{2} \Delta R$, which is permissible provided that in the steady state the ion undergoes simple harmonic motion about its mean position with angular frequency $\omega$. We have thus recast the Ehrenfest equations in terms of the two types of fluctuation in the system represented by $\Delta F$ and $\Delta R$.

Equation 24 above may be integrated to give

$$
\Delta R(t)=\Delta R_{0}(t)-(1 / M \omega) \int_{0}^{t} \Delta F(s) \sin [\omega(s-t)] \mathrm{d} s
$$

where $\Delta R_{0}(t)$ solves $\mathrm{d}^{2} \Delta R_{0} / \mathrm{d} t^{2}=-\omega^{2} \Delta R_{0}$ and describes simple harmonic motion in the adiabatic steady state, defined by $\Delta F=0$. The power into the ion $(w(t))$ is then given by

$$
\begin{aligned}
w(t) & =\frac{\mathrm{d}}{\mathrm{d} t}\left(P^{2} / 2 M+M \omega^{2} \Delta R^{2} / 2\right)=\frac{P(t) \Delta F(t)}{M} \\
& =\frac{P_{0}(t) \Delta F(t)}{M}+\frac{1}{M} \Delta F(t) \int_{0}^{t} \Delta F(s) \cos [\omega(s-t)] \mathrm{d} s
\end{aligned}
$$

where $P_{0}(t)=M \mathrm{~d} \Delta R_{0}(t) / \mathrm{d} t$.

Consider the first term in Eq. (25) given by

$$
w_{0}(t)=\frac{P_{0}(t) \Delta F(t)}{M}
$$

It has the following physical interpretation. Suppose that we drag the ion through the electron gas in a preset motion, described by $\Delta R_{0}(t)$, without allowing the ion to recoil in response to the perturbations that it excites in the gas. The quantity $w_{0}(t)$ then gives the negative of the power dissipated into the electron gas. Physically, we would expect the power dissipated into the electron gas under these conditions to be positive (the ion is doing work on the electron gas), and thus $w_{0}(t)$ to be negative. Therefore, $w_{0}(t)$ describes ionic cooling. 
Consider the second term in Eq. (25), given by

$$
w_{1}(t)=\frac{1}{M} \Delta F(t) \int_{0}^{t} \Delta F(s) \cos [\omega(s-t)] \mathrm{d} s
$$

Its time-average is given by

$$
\begin{aligned}
\left\langle w_{1}\right\rangle & =\frac{1}{M t} \int_{0}^{t} \mathrm{~d} t^{\prime} \Delta F\left(t^{\prime}\right) \int_{0}^{t^{\prime}} \mathrm{d} s \Delta F(s) \cos [\omega(s-t)] \\
& =\frac{1}{2 M t}\left(\int_{0}^{t} \Delta F(s) \cos (\omega s) \mathrm{d} s\right)^{2}+\frac{1}{2 M t}\left(\int_{0}^{t} \Delta F(s) \sin (\omega s) \mathrm{d} s\right)^{2}
\end{aligned}
$$

which is strictly non-negative. We therefore see that $w_{1}$ describes ionic heating.

Thus, the Ehrenfest power has the same underlying structure, with two competing terms, as the power in the classical model of Section 2 and in the quantum model of Section 3. However, the cooling term, $w_{0}$, above is of order $\Delta F$ while the heating term, $w_{1}$, is of order $\Delta F^{2}$. The force $\Delta F$ is produced by the non-adiabatic excitations of the electrons away from the steady state induced by the ionic motion. This in turn is a result of the coupling between the electrons and the ions. Let us characterise the strength of this coupling by the dimensionless small parameter $\lambda$, and let the electron gas start off from the current-carrying steady state, characterised by $\Delta F=0$. Therefore, in the Ehrenfest model the cooling satisfies $w_{0} \propto \lambda$, while the heating satisfies $w_{1} \propto \lambda^{2}$. Consequently the power $w=w_{0}+w_{1}$ is dominated by the cooling term $w_{0}$. In Section 2 we have seen that for classical particles that behave as a fluid we expect heating to be suppressed, but not cooling. Here we have obtained the same result, but now for a quantum fluid. Indeed, we will see below that the dominant term $w_{0}$ in the Ehrenfest power corresponds precisely to the cooling term in the classical model, considered earlier.

\subsection{Classical Approximation}

We now apply the above results to the classical model of Section 2 . The force $F_{e}(t)$ now is a series of delta functions corresponding to individual pointwise electron-ion collisions. The steady state quantity $F_{0}(R)$, on the other hand, now is the time-average of $F_{e}$, with the ion held rigidly at position $R$. Thus, we have now reintroduced a rapidly varying term into the force on the ion. As we will see below, this allows us to recover the heating of the ion and to rederive the analytic classical results in Section 2. To calculate the cooling term $w_{0}$ for the classical model we imagine that the ion sweeps through the jet of classical electrons in a preset motion $\Delta R_{0}$ without recoil. Electron reflections are therefore treated as elastic in the rest frame of the ion. We calculate $\Delta F$ by collecting the resultant momentum transfer, while taking account of the difference in collision frequency depending on the direction of motion of the ion. Substituting into Eq. (26), for the average of $w_{0}$ we recover the cooling term in Eq. (1), $\left\langle w_{0}\right\rangle=4 j(\mathrm{~m} / M)\left(-2 K_{\text {atom }}\right)$.

Now consider the heating term $w_{1}$ in Eq. (27). The force fluctuation $\Delta F(t)=$ $F_{e}-F_{0}$ has a temporal average of zero, with random fluctuations about this average corresponding to the underlying statistical distribution of the arriving classical particles that strike the ion. By a change of integration variable we may write Eq. (27) as

$$
w_{1}=\frac{1}{M} \int_{-t}^{0} \Delta F(t) \Delta F(s+t) \cos (\omega s) \mathrm{d} s
$$


For times $t$ greater than the force-force correlation time, we may approximately write

$$
w_{1}=\frac{1}{M} \int_{-\infty}^{0} \Delta F(t) \Delta F(s+t) \cos (\omega s) \mathrm{d} s
$$

Hence,

$$
\left\langle w_{1}\right\rangle=\frac{1}{2 M} \int_{-\infty}^{\infty} C(s) \cos (\omega s) \mathrm{d} s, C(s)=\langle\Delta F(t) \Delta F(s+t)\rangle
$$

where the angular brackets denote ensemble averaging (which, under the assumption or ergodicity, is equivalent to time-averaging). We see that ionic heating is set by the Fourier component $\omega$ of the force-force correlation function. This is a statement of the fluctuation-dissipation theorem.

Let us evaluate Eq. (28) for our classical model in the limit where the frequency of arrival of the incident particle (equal to the inverse $F-F$ correlation time) far exceeds the frequency of ionic oscillations. In that limit, we may set $\omega=0$ in Eq. (28). We break up time into a sequence of short intervals $\tau$, and give the number of incident particles, $N$, arriving at the ion in any one interval a Poisson distribution with a mean $\langle N\rangle$ and variance $\operatorname{Var}(N)=\left\langle(N-\langle N\rangle)^{2}\right\rangle=\langle N\rangle$. The mean incident particle current is $j=\langle N\rangle / \tau$. The fluctuations in $N$ in different time intervals are uncorrelated. Then $\Delta F$ - the fluctuation of the force, exerted by the incident particles on the ion, away from the mean force $F_{0}$ - in a time interval is $\Delta F=(N-\langle N\rangle)(2 m v) / \tau$, to leading order in the incident particle mass. Replacing the integral in Eq. (28) by a summation over successive intervals then gives

$$
\left\langle w_{1}\right\rangle=\frac{1}{2 M} \operatorname{Var}(N)\left(\frac{2 m v}{\tau}\right)^{2} \tau=4 j\left(\frac{m}{M}\right) K_{e}
$$

Indeed, this is the heating term in Eq. (1), but this time obtained as a consequence of the fluctuation-dissipation theorem.

We may summarise the above observations as follows. The ionic heating originates microscopically from the recoil of the ion in individual electron-ion collisions. In kinetic terms, the heating comes from the high frequency microscopic noise in the force exerted by the electron gas on the ion. This noise, akin to shot noise, is due to the discrete nature of individual collisions. The Ehrenfest approximation neglects these two factors with the result that, in the Ehrenfest model, ionic heating, under exposure to an excited electron gas, is suppressed.

\section{Conclusion}

Three different models that describe the motion of ions in the presence of current carrying electrons have been considered: a purely classical model, a fully quantum model, and a hybrid model in which the electrons are treated as quantum particles and the ions are treated as classical particles. All three models produce one common result, namely that the power delivered to the ions by the electrons contains two competing components, one producing heating and the other producing cooling. The classical and quantum models agree about the degree of heating in the high bias limit, while the hybrid (Ehrenfest) model displays suppressed heating. From the point of view of the quantum model, all transitions involving quantum fluctuations of the ion about its mean position are suppressed in the Ehrenfest approximation because of the tight confinement of the ion to its mean position. However, fluctuations in the mean 
position are still allowed. From the point of view of the classical model the suppression of heating is a consequence of the fact that the electrons are being treated as a smooth fluid rather than a gas of discrete particles. We also find that we expect cooling from a quantum fluid on the basis of a noise analysis.

There is a curious fact in the above that requires further thought. We noted that the Ehrenfest approximation, in which the ions are highly localised and behave classically, prevents heating by the mechanism described by the quantum perturbation formalism (quantum fluctuation of the ions about their mean positions). At the same time we observed that the fully classical simulations, in which both the ions and the electrons obey classical equations of motion, produced heating in agreement with the quantum formalism. Clearly in the classical case there are no quantum fluctuations of the ions about their mean positions, but there are classical fluctuations. (In the Ehrenfest method even the classical fluctuations are suppressed.) This raises the intriguing question: what is the connection between classical fluctuations and quantum fluctuations? We offer no answer to this question, but merely observe that these results suggest that a natural route from quantum to classical mechanics might be through a hidden variable method. This type of approach, based on Bohm-de Broglie dynamics, has been considered in the context of electron scattering[22].

Finally we note that we have offered a suggestion for a scheme to reintroduce correlations between electrons and the ions, which is currently being investigated.

\section{Acknowledgments}

DRB gratefully acknowledges the Royal Society for funding through a Research Fellowship. TNT gratefully acknowledges support for this work from EPSRC under grant number GR/R36077. We would like to thank Adrian Sutton for his critical reading of the manuscript and numerous important conversations.

[1] Brandbyge M, Mozos J L, Ordejon P, Taylor J, and Stokbro K 2002 Phys. Rev. B 65(16) 165401

[2] Di Ventra M and Lang N D 2002 Phys. Rev. B 65(4) 45402

[3] Di Ventra M and Pantelides S T 2000 Phys. Rev. B 61(23) 16207

[4] Landauer R 1989 J. Phys.: Condens. Matter 18099

[5] Todorov T N, Hoekstra J, and Sutton A P 2000 Phil. Mag. B 80421

[6] Todorov T N, Hoekstra J, and Sutton A P 2001 Phys. Rev. Lett. 863606

[7] Todorov T N 2002 J. Phys.: Condens. Matter 143049

[8] Stoneham A M 1981 Rep. Prog. Phys. 441251

[9] Gersonde I H and Gabrial H 1993 J. Chem. Phys. 98(3) 2094

[10] Krylov A I and Gerber R B 1997 J. Chem. Phys. 106(16) 6574

[11] Margulis C J and F. C D 1997 J. Chem. Phys. 106(16) 6574

[12] Todorov T N 1998 Phil. Mag. B 77(4) 965

[13] Montgomery M J, Todorov T N, and Sutton A P 2002 J. Phys.: Condens. Matter 145377

[14] Chen Y C, Zwolak M, and Di Ventra M 2003 Nano. Lett. 31691

[15] Horsfield A P, Bowler D R, and Fisher A J 2004 J. Phys.: Condens. Matter 16 L65

[16] Montgomery M J and Todorov T N 2003 J. Phys.: Condens. Matter 158781

[17] Todorov T N 2001 J. Phys.: Condens. Matter 1310125

[18] Calvayrac F, Reinhard P G, Suraud E, and Ullrich C A 2000 Physics Reports 337(6) 493

[19] Tomfor J K and Sankey O F 2001 Phys. Stat. Sol.(B) 226(1) 115

[20] Mita K 2001 Am. J. Phys. 69(4) 470

[21] Käb G 2002 Phys. Rev. E 66(4) 046117

[22] Gindensperger E, Meier C, and Beswick J A 2002 J. Chem. Phys. 116(1) 8 